\title{
The a7 Nicotinic Agonist ABT-I 26 in the Treatment of Cognitive Impairment Associated with Schizophrenia in Nonsmokers: Results from a Randomized Controlled Phase 2b Study
}

\author{
George Haig*,', Deli Wang', Ahmed A Othman',2 and Jun Zhao' \\ 'Abbvie Inc., North Chicago, IL, USA; ' ${ }^{2}$ aculty of Pharmacy, Cairo University, Cairo, Egypt
}

\begin{abstract}
A double-blind, placebo-controlled, parallel-group, 24-week, multicenter trial was conducted to evaluate the efficacy and safety of 3 doses of ABT-126, an $\alpha 7$ nicotinic receptor agonist, for the treatment of cognitive impairment in nonsmoking subjects with schizophrenia. Clinically stable subjects were randomized in 2 stages: placebo, ABT-126 25 mg, $50 \mathrm{mg}$ or $75 \mathrm{mg}$ once daily (stage I) and placebo or ABT-I $2650 \mathrm{mg}$ (stage 2). The primary analysis was the change from baseline to week 12 on the MATRICS Consensus Cognitive Battery (MCCB) neurocognitive composite score for ABT- $12650 \mathrm{mg}$ vs placebo using a mixed-model for repeated-measures. A key secondary measure was the University of California Performance-based Assessment-Extended Range (UPSA-2ER). A total of 432 subjects were randomized and $80 \%(344 / 431)$ completed the study. No statistically significant differences were observed in either the change from baseline for the MCCB neurocognitive composite score (+2.66 [ \pm 0.54$]$ for ABT- $12650 \mathrm{mg}$ vs $+2.46[ \pm 0.56]$ for placebo at week 12; $P>0.05)$ or the UPSA-2ER. A trend for improvement was seen at week 24 on the 16-item Negative Symptom Assessment Scale total score for ABT- $12650 \mathrm{mg}$ (change from baseline $-4.27 \pm[0.58]$ vs $-3.00 \pm[0.60]$ for placebo; $P=0.059$ ). Other secondary analyses were generally consistent with the primary end point results. Adverse event rates were similar for ABT- 126 and placebo. ABT- 126 did not demonstrate a consistent effect on cognition in nonsmoking subjects with schizophrenia; however, a trend toward an effect was observed on negative symptoms. ClincalTrials.gov registration: NCT0 655680.

Neuropsychopharmacology (2016) 4I, 2893-2902; doi: I0.1038/npp.2016.101; published online 10 August 2016
\end{abstract}

\section{INTRODUCTION}

Nearly all patients with schizophrenia are compromised in their ability to function in the community due to cognitive impairment (Keefe et al, 2005). This is in addition to the effects associated with positive, negative, and mood symptoms. Whereas the severities of other core symptoms of the disease vary and are typically episodic, cognitive impairment is persistent throughout the course of illness, with a fairly consistent level of severity. Cognitive impairment commences prior to the onset of the other symptom domains and, with the exception of focused remediation therapy, is not amenable to treatments in the long term (Bowie and Harvey, 2005; Green et al, 2004).

*Correspondence: Dr G Haig, AbbVie Inc., R48B/Bldg. AP4- I, I North Waukegan Rd., North Chicago, IL 60064, US, Tel: +847 938 1900, Fax: +847 937 0745, E-mail: george.haig@abbvie.com

Previously presented at the International Congress on Schizophrenia Research, Colorado Springs, CO March 28-April I, 20I5. Work was performed at AbbVie Inc., I North Waukegan Rd., North Chicago, IL 60064, USA.

Received 9 March 2016; revised 23 May 2016; accepted I2 June 2016; accepted article preview online 20 June 2016
The nicotinic acetylcholine receptor (nAChR) system has been studied as a possible target to improve cognition in schizophrenia and other cognitive disorders. The procognitive effects of nicotine have been known for some time; however, addictive properties, short duration of action, and development of tachyphylaxis limit its utility as a therapeutic agent (Harris et al, 2004). Of the possible nAChR targets, the $\alpha 7$ receptor has been one of particular interest in terms of drug development for the treatment of schizophrenia-related cognitive impairment. People with schizophrenia have decreased expression of brain $\alpha 7 \mathrm{nAChRs}$, and these deficits are most prominent in the hippocampus, frontal cortex, and thalamus (Freedman et al, 1995; Guan et al, 1999; Levin and Rezvani, 2007). The decreased expression of $\alpha 7$ receptors has led to speculation that smoking is self-medication for cognitive impairment (Levin and Rezvani, 2007).

A number of $\alpha 7 \mathrm{nAChR}$ agonists have been developed and tested in the clinic, including GTS-21, encenicline, TC-5619, and RG3487, with positive or mixed results (Keefe et al, 2015; Lieberman et al, 2013; Olincy et al, 2006; Umbricht et al, 2014). A possible interaction between nicotinic receptor ligands such as $\alpha 7$ agonists and nicotine has been observed. First reported by Freedman et al (1995), it was thought that 
chronic exposure to nicotine results in nicotinic receptor desensitization (Freedman et al, 1995). Many of the trials involving nicotinic compounds either excluded patients who smoke or, if allowed to participate, required smoking restrictions during the cognition testing period (AhnAllen et al, 2008; Freedman et al, 2008; Harris et al, 2004; Myers et al, 2004; Smith et al, 2006).

ABT-126 is a potent and selective $\alpha 7 \mathrm{nAChR}$ agonist that has exhibited high affinity for the $\alpha 7 \mathrm{nAChR}$ in nonclinical studies, and demonstrated efficacy in animal models relevant to cognitive impairment associated with schizophrenia. ABT-126 was well tolerated in phase 1 studies, has a pharmacokinetic half-life that supports once-daily dosing, and is virtually devoid of drug-drug interaction liabilities. We have previously reported the results of a 12-week, placebocontrolled study of ABT-126 in 207 subjects with schizophrenia who were clinically stable (Haig et al, 2016). In the overall analysis, a dose-related trend toward an effect of ABT-126 on cognitive improvement was observed. In a subgroup analysis by smoking status, we observed that ABT-126 10 and $25 \mathrm{mg}$ once daily had an apparent doserelated effect on the primary end point, the Measurement and Treatment Research to Improve Cognition in Schizophrenia (MATRICS) Consensus Cognitive Battery (MCCB) (Kern et al, 2008; Nuechterlein et al, 2008) composite score, in nonsmokers, with a Cohen's d effect size of $>0.80$ in the highest dose group. In smokers, there was no treatment effect observed at any dose. A trend toward significance was also noted among nonsmokers on the University of California San Diego Performance-based Skills Assessment (UPSA-2) (Patterson and Goldman, 2005).

As a follow-up to that study, we conducted a dose-ranging study in nonsmokers with schizophrenia that utilized higher ABT-126 doses to better understand the dose-response relationship and assess the durability of effect. Estimates on smoking rates in the schizophrenia population vary, but the consensus is that the majority of them smoke (de Leon and Diaz, 2005). Nevertheless, a treatment to improve cognition in a subset of the patient population would be considered a major advancement.

\section{MATERIALS AND METHODS}

\section{Study Design}

The objective of this study was to evaluate the efficacy and safety of ABT-126 in treating cognitive impairment in subjects with schizophrenia who were clinically stable, receiving antipsychotic medications, and did not currently smoke. Protocols, amendments, and informed consent forms were approved by institutional review boards and independent ethics committees. Written informed consent was obtained before any study procedures were initiated. The MATRICS guidelines (Buchanan, 2006; Buchanan et al, 2005) were considered in the design and conduct of this study. The study was conducted from May 2012 to July 2014 at 34 sites in the United States (US), Great Britain, and Russia.

The study consisted of a screening and prospective stabilization period lasting 28 to 42 days prior to randomization, a 24-week treatment period, and a 14-day follow-up period after the last dose of study drug (Supplementary
Figure S1). Subjects returned at weeks $1,2,4,6,8,10,12,14$, $16,18,20,22$, and 24 . There were 2 stages of randomization in the study. During stage 1, 279 subjects were randomized in a $1: 1: 1: 1$ ratio to ABT-126 $25 \mathrm{mg}, 50 \mathrm{mg}, 75 \mathrm{mg}$, or matching placebo. Using pre-established decision rules that considered estimated effect sizes and their predictive probabilities of success as well as safety/tolerability for each dose group, an independent, unblinded efficacy data monitoring committee (DMC) used interim data to select the dose of ABT-126 for use in stage 2 of the study. An additional 153 subjects were subsequently randomized in a $1: 1$ ratio to ABT-126 or placebo. Randomization was conducted via an interactive voice-response/web-based system using a randomization schedule provided by the sponsor.

\section{Subjects}

Eligible subjects were men and women between 20 and 65 years of age with a current diagnosis of schizophrenia who were receiving stable doses of 1 to 2 allowed antipsychotics. Subjects were clinically stable and had a diagnosis of schizophrenia for $\geqslant 2$ years prior to screening. Stability criteria included no psychiatric inpatient hospitalization or destabilization within the previous 4 months, no symptomrelated changes in psychotropic medications within 8 weeks prior to baseline, no changes in medications within 4 weeks prior baseline, core positive symptoms no worse than moderate in severity, extrapyramidal symptoms (EPS) no worse than mild in severity, and no evidence of a major depressive episode. Eligible subjects were current nonsmokers, meaning they had not smoked on a regular basis for $\geqslant 3$ months. Nonsmoking status was confirmed with urine cotinine tests prior to and at several visits following randomization. Participants were otherwise healthy based on their medical history, physical examination, vital signs, laboratory testing, and a 12-lead electrocardiogram (ECG). Subjects with potential or known risk factors for Torsades de Pointes, a clinically significant abnormal ECG, or an ECG with a prolonged QT interval were excluded. Other major exclusion criteria included a history of substance abuse, evidence of suicidality or violent ideation, hepatitis or HIV infection, and body mass index (BMI) $>45 \mathrm{~kg} / \mathrm{m}^{2}$ or body weight $>145 \mathrm{~kg}$.

Participants remained on their baseline antipsychotic regimen during the entire study. Most typical and atypical antipsychotics were permitted. Anticholinergics were allowed during the study; however, they had to be at stable doses and were not to be taken within 12 hours prior to protocol-specific cognitive testing. Mood stabilizers, sertindole, iloperidone, chlorpromazine, pimozide, thioridazine, citalopram > $20 \mathrm{mg}$ daily, bupropion, highly anticholinergic tricyclic antidepressants, or monoamine oxidase inhibitors were excluded. Hypnotic doses of clozapine $(\leqslant 100 \mathrm{mg})$ were allowed.

\section{Primary Efficacy}

The primary efficacy measure was the MCCB neurocognitive composite score change from baseline to week 12 . The MCCB neurocognitive score contains all of the tests and domains of the MCCB composite score with the exception of 
social cognition. In addition to having good test-retest reliability, the MCCB discriminates patients with schizophrenia from normal subjects and correlates with functional status (Kern et al, 2008; Nuechterlein et al, 2008). The MCCB was conducted during screening, at baseline (day -1$)$, and at weeks $6,12,18$, and 24 . The decision to use the MCCB neurocognitive composite score as the primary end point, rather than the MCCB composite score, was due to greater sensitivity being shown with the former in a previous study (Haig et al, 2016). This change was made via protocol amendment.

\section{Secondary Efficacy}

Secondary cognitive and functional measures included the MCCB composite and domain scores, the UPSA-2 Extended Range total score (UPSA-2ER), and the Schizophrenia Cognition Rating Scale (Keefe et al, 2006) (SCoRS) total score and global rating scale. The UPSA-2ER is a modified version of the UPSA-2 (Patterson and Goldman, 2005) created for the current study. All of the tasks and domains (Organization/Planning, Financial Skills, Communication, Transportation, Household Management, and Medication Management) of the UPSA-2 are retained in the UPSA-2ER, with the addition of 1 or 2 tasks in each domain that are intended to make the test more challenging and to reduce possible ceiling effects. An interview-based measure of community functioning, the SCoRS, was administered at weeks 10 and 22; weeks 12 and 24 were avoided on the SCoRS to reduce visit burden. NeuroCog Trials Inc. (Chapel Hill, NC) provided rater training and quality assurance for the MCCB, UPSA-2ER, and SCoRS.

Another secondary efficacy measure, the Negative Symptom Assessment Scale (NSA-16) (Alphs et al, 1989), is a 16-item instrument plus a 1-item global rating designed to measure the severity of specific negative symptoms in schizophrenia. The NSA-16 was conducted at baseline and weeks $6,12,18$, and 24 . Subjects' schizophrenia symptoms were assessed throughout the study using the Positive and Negative Syndrome Scale (Kay et al, 1987) (PANSS) and the Clinical Global Impression-Severity (CGI-S) (Guy, 1976).

\section{Safety, Tolerability, and Pharmacokinetics}

Safety was assessed through regular physical examinations, clinical laboratory tests, 12-lead ECGs, and vital sign measurements. Clinical symptoms were assessed regularly using the Columbia Suicide-Severity Rating Scale (C-SSRS) (Posner et al, 2011), Abnormal Involuntary Movement Scale (AIMS) (Guy, 1976), Barnes Akathisia Scale (BAS) (Barnes, 1989), Simpson Angus Scale (SAS) (Simpson and Angus, 1970), and the Physician Withdrawal Checklist-20 (PWC-20) (Rickels et al, 2008) during follow-up.

A blood sample for pharmacokinetic analysis was collected during each visit at weeks 2, 4, 6, 10, 12, 16, 18, 22, and 24 .

\section{Statistical Analysis}

A total of 430 subjects were planned for this study. The planned sample size for stage 1 (70/arm, total 280) allowed $>85 \%$ power to detect an effect size of 0.5 comparing an ABT-126 dose group with placebo for change from baseline to week 12 on the MCCB neurocognitive composite score at a 1 -sided significance level of 0.05 . At the completion of the study, stage 1 data for the ABT-126 $50 \mathrm{mg}$ dose and placebo groups were combined with their respective stage 2 counterparts. The additional stage 2 sample size of 150 allowed the study to have $80 \%$ power to detect an effect size of 0.30 for the change from baseline to final assessment in UPSA-2ER total score when comparing the ABT-126 $50 \mathrm{mg}$ dose group with placebo. This calculation assumed a 1-sided significance level of 0.05 and that $10 \%$ of subjects would have no data after randomization. The 1-sided test was chosen because ABT-126 was being studied to demonstrate improvement vs placebo. No adjustments for multiplicity were made.

Two analysis sets were defined from the intent-to-treat (ITT) data set: the primary set (all subjects taking placebo or ABT-126 $50 \mathrm{mg}$ ) and the dose-ranging set (randomized subjects from stage 1 ). The primary efficacy end point was the change from baseline to week 12 in the MCCB neurocognitive composite score in the primary set. The primary analysis was a likelihood-based, mixed-effects model, repeated measures (MMRM) analysis of the change from baseline to each post-baseline assessment up to and including week 24. The model included fixed, categorical effects for treatment, site, visit, and treatment-by-visit interaction, with continuous fixed covariates for baseline score and the baseline score-by-visit interaction. An unstructured covariance structure was used to model the within-patient correlation. As a secondary analysis, the same MMRM analysis was performed on the MCCB neurocognitive composite scores in the dose-ranging set as well as on the other efficacy measures.

Secondary analyses on the primary and secondary efficacy variables were also performed using an analysis of covariance (ANCOVA) model with factors of treatment and site, and with baseline score as a covariate for the change from baseline to final evaluation in MCCB up to weeks 12 and 24 in both ITT subsets. Statistical analyses for efficacy assessments were 1-sided, with $P<0.10$ indicating a trend and $P<0.05$ indicating statistical significance.

Subgroup analyses were conducted on the MCCB composite, neurocognitive composite, and domain score changes from baseline to last observation up to week 12 and 24 to see if gender, age group ( $\leqslant 40 v s>40$ years), or study site had an effect on treatment response. Post hoc exploratory analyses for MCCB, NSA-16, and UPSA-2ER included subgroup analyses of subjects enrolled in the US or Russia. Safety analyses were performed on the safety data set, which included all subjects who received $\geqslant 1$ dose of study drug. Statistical tests for safety variables were 2 -tailed at $\alpha=0.050$, if applicable.

\section{RESULTS}

\section{Subject Disposition and Baseline}

A total of 279 subjects were randomized to ABT-126 $25 \mathrm{mg}$, $50 \mathrm{mg}, 75 \mathrm{mg}$, or placebo in stage 1 . Based on a prespecified analysis of interim data, the independent efficacy DMC selected ABT-126 $50 \mathrm{mg}$ as the dose to study in stage 2 (in which patients were treated with either ABT-126 $50 \mathrm{mg}$ or placebo), and another 153 subjects were randomized. One subject was excluded from the ITT population because no 


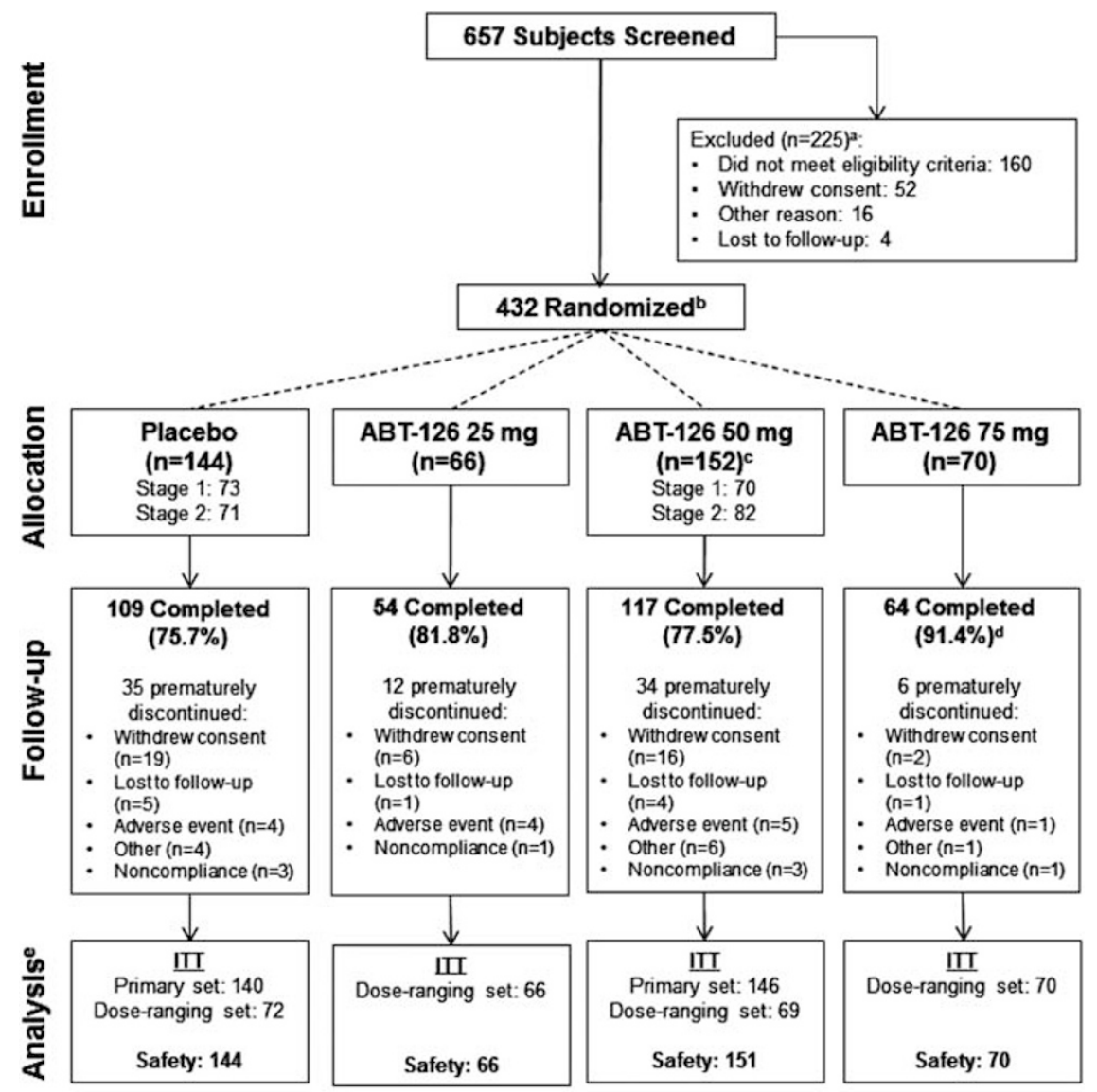

Note: The primary reason for discontinuation is provided for subjects who prematurely discontinued $\mathrm{ITT}=$ intent-to-treat

a Subjects could have more than one reason for exclusion

211 in the United States, 208 in Russia, 13 in the United Kingdom

-1 subject was randomized but did not take study drug

dP $=0.033$ for any reason for discontinuation vs. all other treatment groups

e 10 subjects were excluded from efficacy analyses due to possible data falsification at 1 clinical site $(n=9)$ and did not take study drug $(n=1)$

Figure I Subject disposition.

study drug was received. A total of $344 / 431$ subjects $(79.8 \%)$ completed the study. Subject disposition is illustrated in Figure 1, and a summary of baseline characteristics is presented in Table 1. Risperidone (28.3\%), quetiapine (20.6\%), aripiprazole (14.8\%), haloperidol (14.6\%), and olanzapine $(11.4 \%)$ were the most commonly used concomitant antipsychotic medications.

\section{Medication Compliance}

Overall, $88.4 \%$ of subjects were considered compliant with study drug based on capsule counts of returned blister cards. No statistically significant differences among treatment groups were observed for compliance at any visit or for overall compliance. Pharmacokinetic analysis of ABT-126 plasma concentrations confirmed pill count assessment of compliance. At week 12 using the sparse pharmacokinetic samples collected in the study, the mean ABT-126 plasma concentrations for subjects with samples collected 3 to 6 hours post-dose (approximate maximum plasma concentration) and 18 to 30 hours post-dose (approximate trough concentration) were $22.3 \pm 14.6$ and $15.8 \pm 11.5 \mathrm{ng} / \mathrm{ml}$, respectively, for the $25 \mathrm{mg}$ dose; $45.0 \pm 27$ and $35.3 \pm 31.8 \mathrm{ng} /$ $\mathrm{ml}$, respectively, for the $50 \mathrm{mg}$ dose; and $75.6 \pm 41.4$ and $51.9 \pm 26.6 \mathrm{ng} / \mathrm{ml}$, respectively, for the $75 \mathrm{mg}$ dose. These results were consistent among the different study visits, and the mean ABT-126 plasma concentrations in each dose group were aligned with predicted concentrations based on previous studies of ABT-126 (with consideration for the higher variability due to the phase 2 setting and wider sample collection windows).

\section{Efficacy}

No statistically significant improvement in the primary end point was seen in either the primary or dose-finding sets (Figure 2). Similarly, no statistically significant difference was observed between ABT-126 and placebo treatment for the functional end point, the UPSA-2ER (Figure 3). MMRM analyses of UPSA-2ER results, however, showed trends for 
Table I Baseline Characteristics ${ }^{a}$

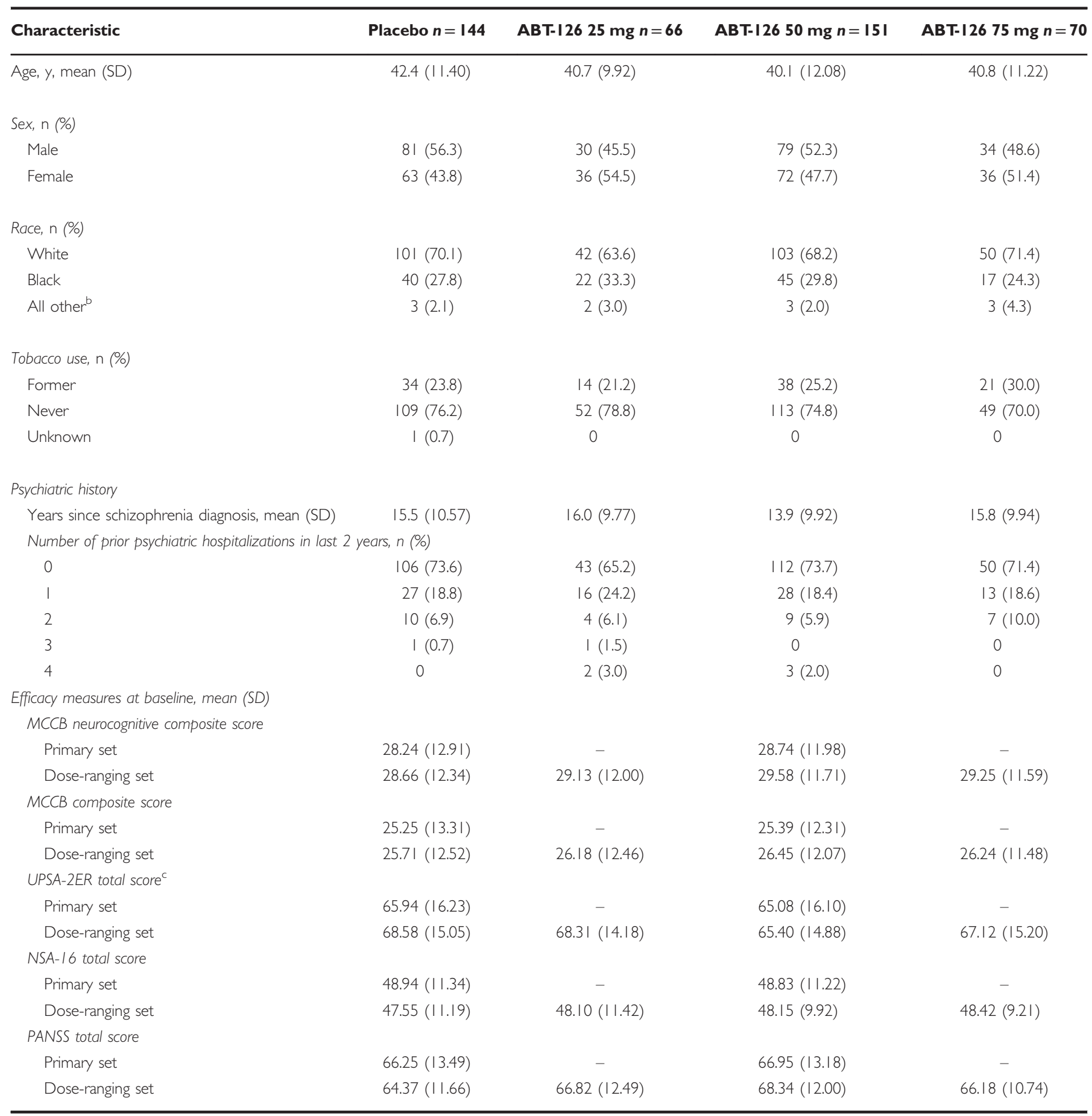

Abbreviations: MCCB, MATRICS Consensus Cognitive Battery; NSA-16, 16-item version of the Negative Symptom Assessment scale; PANSS, Positive and Negative Symptom Scale; SD, standard deviation; UPSA-2ER, University of California San Diego Performance-based Skills Assessment-2 Extended-Range.

a Safety dataset unless otherwise stated.

bIncludes Asian, multi-race, and Hawaiian native.

'Excludes the medication management subscale as it is not included in the ex-US version.

an overall treatment effect $(P=0.088)$ and a greater improvement with ABT-126 $50 \mathrm{mg}$ vs placebo at week 12 $(P=0.095$; Figure 3$)$ in the primary set. A trend toward significance in NSA-16 total score change from baseline was observed with ABT-126 $50 \mathrm{mg}$ at week 24 in the primary set
$(P=0.059)$ and for the $50 \mathrm{mg}$ and $75 \mathrm{mg}$ dose groups at week 24 in the dose-ranging set $(P=0.056$ and $P=0.078$, respectively; Figure 3).

A significant difference in SCoRS total score compared with placebo was observed at week 22 for ABT-126 $50 \mathrm{mg}$ in 
a

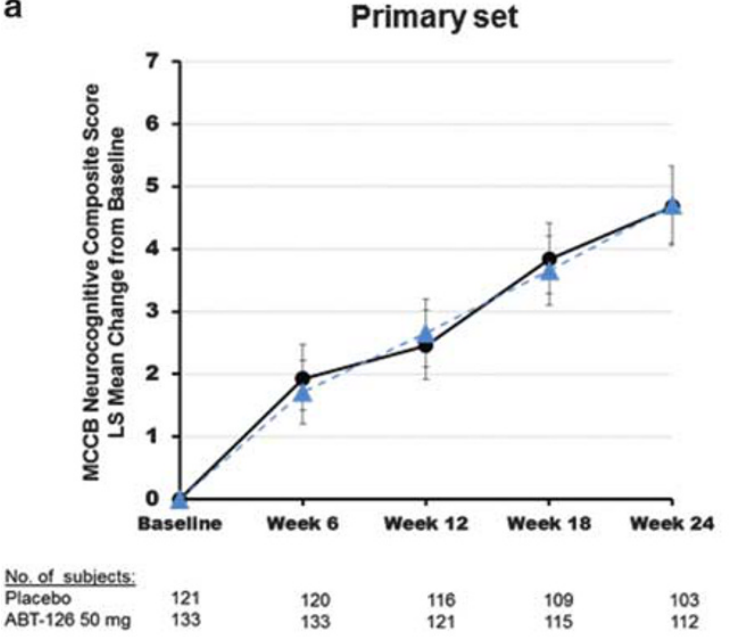

b

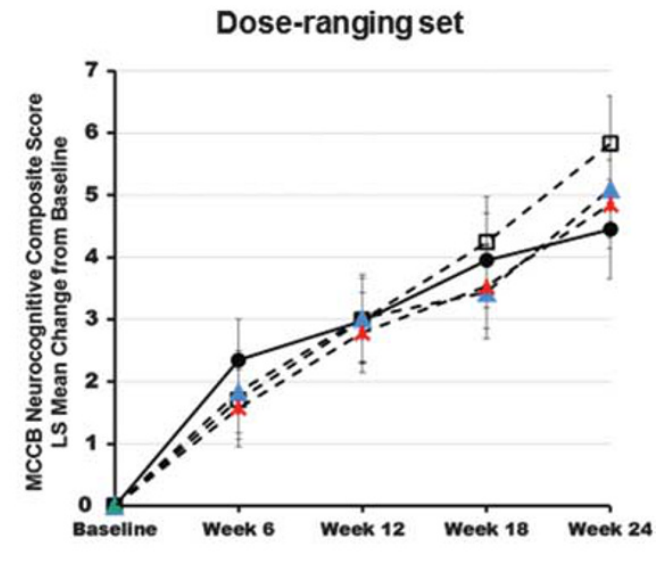

No. of subjects:
Placebo
ABT-126 $25 \mathrm{mg}$

ABT- $12625 \mathrm{mg}$
ABT-126 $50 \mathrm{mg}$ ABT-126 $50 \mathrm{mg}$
ABT-126 $75 \mathrm{mg}$

—Placebo - - ABT-126 $25 \mathrm{mg}-\mathrm{A}$ - ABT-126 $50 \mathrm{mg} \longrightarrow$ - ABT-126 $75 \mathrm{mg}$

Figure 2 LS Mean change from baseline in MCCB neurocognitive composite total score. Error bars represent the standard error of the LS means. An increase in score indicates improvement. There were no statistically significant I-sided p-values from the repeated measures model. The model included treatment, site, visit, baseline score, interactions of treatment and visit, and interaction of baseline score and visit; covariance structure was unstructured. LS, least squares; MCCB, MATRICS Consensus Cognitive Battery.

the primary set $(P=0.004)$, and a trend was noted in the global rating scale $(P=0.054$; Supplementary Figure S2). In the dose-ranging set, a treatment effect was only observed for the SCoRS total score $(P=0.022)$. Similar findings were observed using ANCOVA (data not shown). Changes from baseline in GCI-S score indicated no deterioration in subjects' severity of overall illness during the study. Decreases (indicating improvement) in PANSS score were observed with active treatment, but the differences vs placebo were not statistically significant for any ABT-126 dose group. Results from the ANCOVA analyses for CGI-S and PANSS in the primary set are presented in Supplementary Table S1.

\section{Subgroup Analyses}

Post hoc exploratory analyses indicated regional differences on most efficacy measures. ABT-126-treated US subjects generally had greater treatment effects compared with placebo than ABT-126-treated subjects enrolled in Russia, where larger practice (data not shown) and placebo effects (Supplementary Table S2) were observed. For the primary end point, $\mathrm{MCCB}$ neurocognitive composite score, a trend for a treatment effect $v s$ placebo was observed among US subjects treated with ABT-126 $50 \mathrm{mg}$ in the primary set (overall $P=0.082$; Supplementary Table S2) and with ABT-126 $75 \mathrm{mg}$ at week 24 in the dose-ranging set $(P=0.079$; data not shown). Trends for greater improvement in UPSA-2ER with ABT-126 $50 \mathrm{mg}$ vs placebo were also observed in the subgroup of US subjects in the primary set $(P=0.084$ at week 12 and $P=0.088$ overall; Supplementary Table S2). NSA-16 total score improvement was significant in the US study population overall and at various time periods in the primary set $(P<0.05$; Supplementary Table S2), with notable treatment effects on the social activity and global negative symptom subscales at week 24 in the primary set $(P=0.002$ and $P=0.034$, respectively; data not shown). Among study participants from Russia, differences in least-squares (LS) mean change from baseline between ABT-126 treatment vs placebo were not statistically significant for MCCB neurocognitive composite score, UPSA-2ER, or NSA-16 (Supplementary Table S2).

Subgroup analyses did not reveal any effect on treatment response (as measured by the MCCB composite, neurocognitive composite, or domain scores) based on gender, age group ( $\leqslant 40$ versus $>40$ years), or study site.

\section{Safety}

Adverse events are summarized by treatment group in Table 2 . There was no statistically significant difference in the overall incidence of adverse events among treatment groups. Incidences of insomnia ( $7.1 \%$ vs $1.4 \%, P=0.039)$ and fatigue ( $4.3 \%$ vs $0 \% ; P=0.034)$ were both significantly higher for ABT-126 $75 \mathrm{mg}$ vs placebo.

One subject from the ABT-126 $25 \mathrm{mg}$ group died 15 days after the end of treatment due complications of hypertensive cardiovascular disease and emphysema. The death was assessed as not related to study drug. Overall, no clinically meaningful changes were noted on physical exam, clinical laboratory, ECG, C-SSRS, movement rating scales (SAS, BAS, AIMS), or PWC-20 assessments.

A long-term, open-label extension to this study was initiated, but was terminated prior to completion when the main study failed to meet the primary end point. Patient disposition from the extension study and a summary of the safety findings are shown in Supplementary Figure S3 and Supplementary Table S3, respectively. No new safety signals were detected in this study. 
a

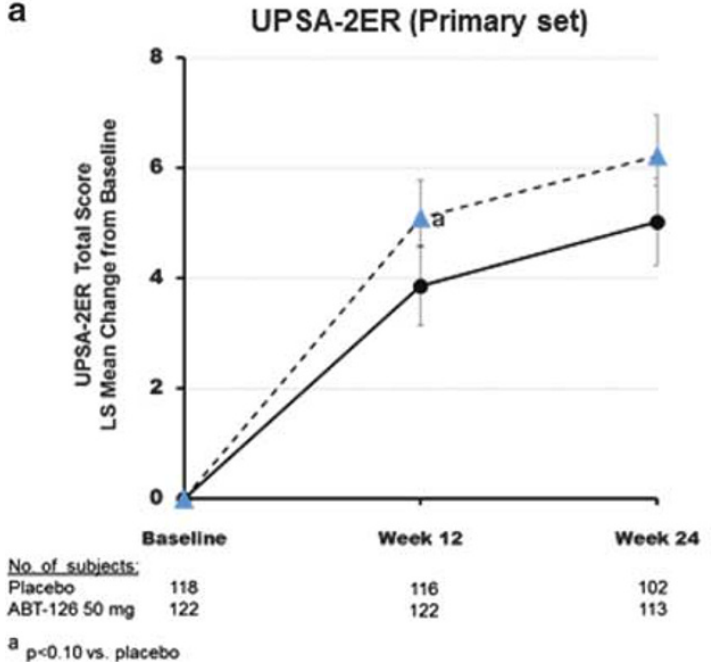

c

NSA-16 (Primary set)

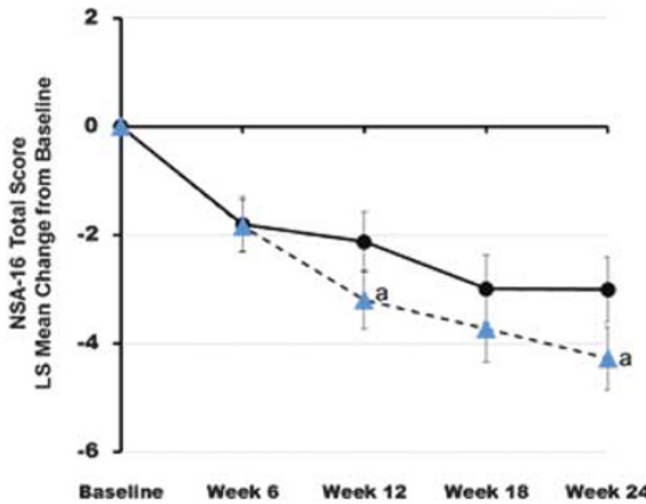

№ of subjects:

No of subie Alacebo $12650 \mathrm{mg}$

Baseline

Week 6

Week 12

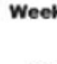

$a_{p<0.10 \mathrm{vs} \text {. placebo }}$

\section{Placebo}

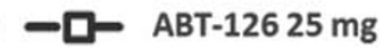

b

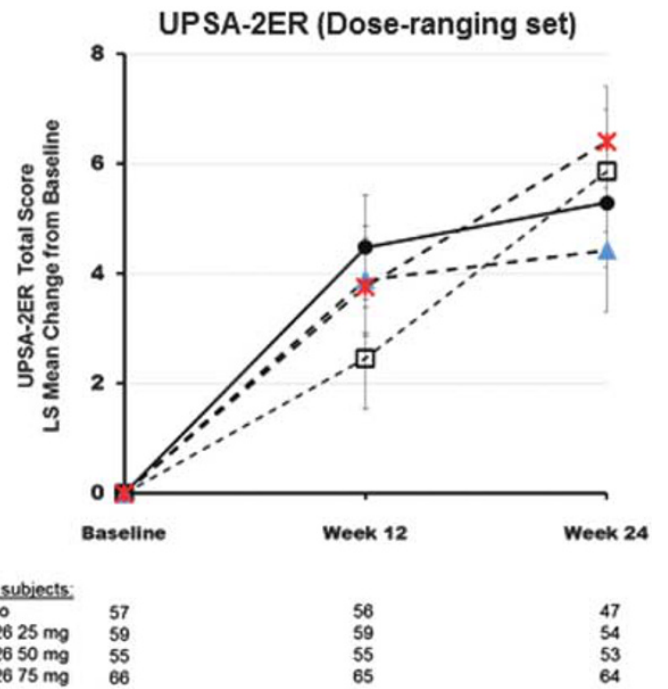

d

NSA-16 (Dose-ranging set)

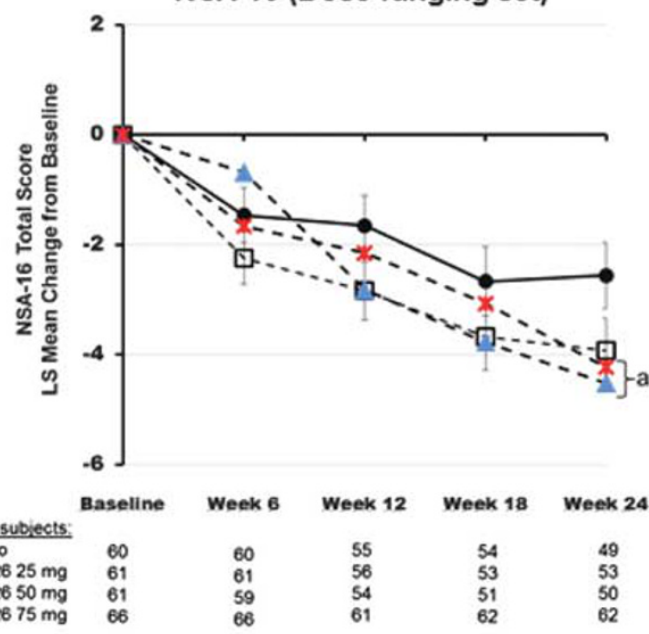

Figure 3 LS mean change from baseline in UPSA-2ER and NSA- 16 total scores. The repeated measures model included treatment, site, visit, baseline score, interactions of treatment and visit, and interaction of baseline score and visit; covariance structure was unstructured. There were no statistically significant treatment effects from the repeated measures model analysis in the UPSA-2ER or NSA-16. The LS mean change from baseline on the UPSA-2ER total score in the primary set (a) and dose-ranging set (b). An increase in score indicates improvement and error bars represent the standard error of the LS means. The UPSA-2ER total score depicted in this graph does not include the medication management subscale score because the ex-US version does not use the medication management test. The range of the UPSA-2ER total score without the medication management subscale is 0 to I00. The LS mean change from baseline on the NSA-16 total score in the primary set (c) and dose-ranging set (d). A decrease in score indicates improvement and error bars represent the standard error of the LS means. LS, least squares; NSA-16, I6-item Negative Symptom Assessment Scale; UPSA-2ER, University of California San Diego Performance-based Skills Assessment-2 Extended Range.

\section{DISCUSSION}

This study was conducted to determine whether the effect observed in the nonsmoking subgroup in a previous multicenter study with ABT-126 could be reproduced. Despite the wider dose range evaluated in this study, ABT-126 did not demonstrate a consistent treatmentrelated effect on cognition as measured by the МССВ neurocognitive composite score in a population of clinically stable, nonsmoking subjects with schizophrenia.

Of the nearly 450 subjects enrolled, approximately half were from the US and half were from Russia. Only a limited number of subjects were enrolled from sites in the UK. This enabled some interesting regional analyses, which revealed that subjects in Russia consistently had much higher practice effects and placebo responses on nearly all end points. To limit the influence of practice effects, the first of the cognition tests were administered prior to randomization. In addition, an experienced vendor was used to train and monitor the administration and scoring of all scales. Our previous experience with the MCCB indicated a low to modest practice effect of 1 to 2 points in 3-month studies.

There are several possible reasons for the apparent regional inconsistencies. First, the mean age and duration 
Table 2 Summary of Adverse Events

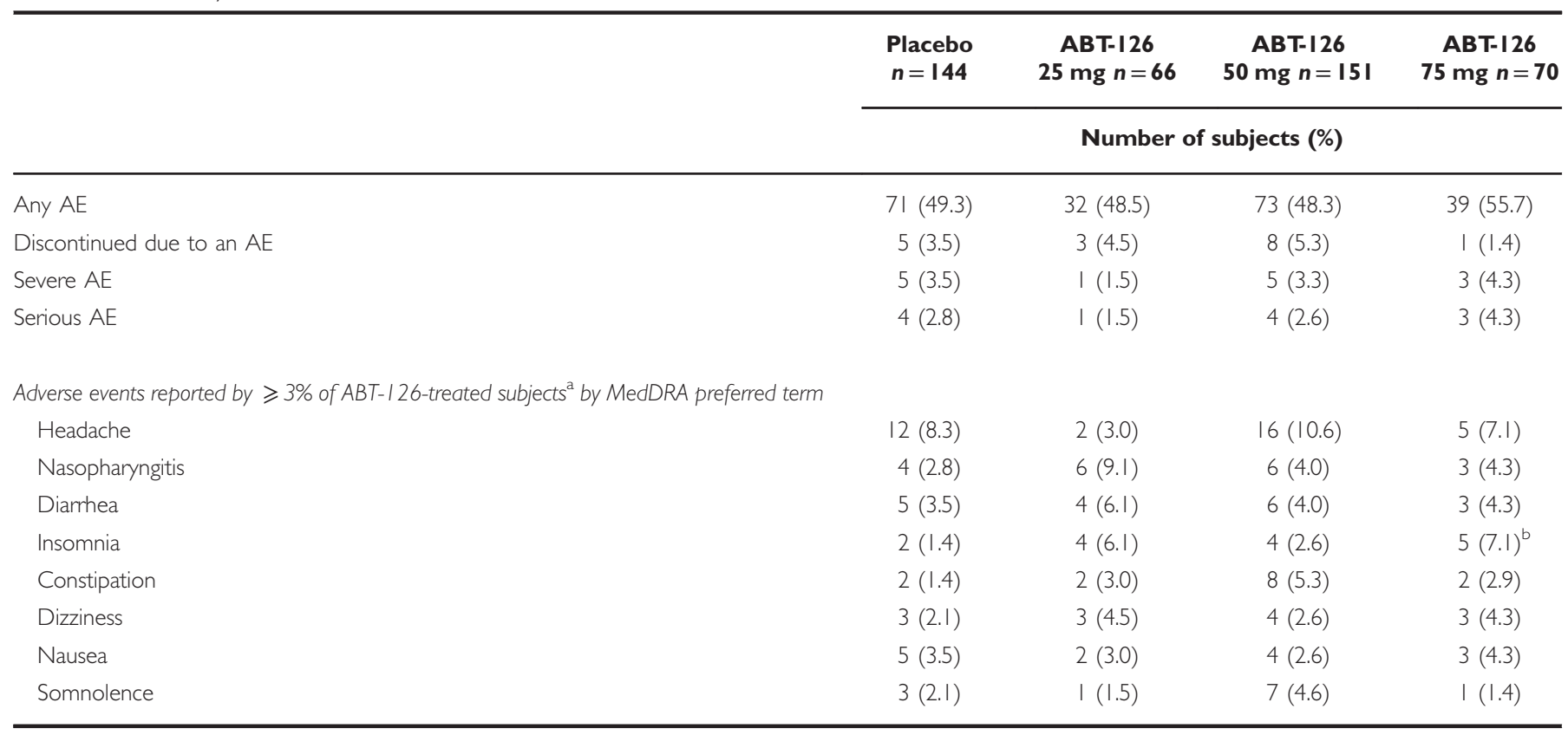

Abbreviation: MedDRA, Medical Dictionary for Regulatory Activities.

${ }^{a}$ All ABT- 126 treatment groups combined.

${ }^{\mathrm{b}} \mathrm{P}<0.05$ vs placebo.

of illness were significantly higher among US vs Russian subjects (data not shown; $P<0.001$ for the comparison). Investigators have reported that younger age and shorter duration of illness contribute to a heightened placebo response in antipsychotic medication clinical studies (Agid et al, 2013; Dold and Kasper, 2015). It has also been observed that younger patients with schizophrenia are more responsive to cognitive remediation therapy (Kontis et al, 2013), whereas older patients show reduced practice effects (Granholm et al, 2010). Notably, ABT-126 plasma concentrations were comparable for subjects enrolled in Russia and the US (data not shown), suggesting that the regional differences are not related to ABT-126 exposure. The second possible explanation is that naive subjects (in terms of exposure to cognition tests), such as those enrolled in Russia, have a higher learning curve on cognition tests, and we observed the ascending portion of the learning curve. This explanation, however, does not account for the high placebo response in Russia on scales such as the NSA-16 and the SCoRS, which are not prone to practice effects. Lastly, the observed differences may be due to the visit structure of the study. Subject visits were required every 2 weeks, which was advocated by US investigators to improve medication and overall study compliance, but may have led to a form of remediation. We learned that health care professional contact with the schizophrenia patient population is rather limited in many Eastern European countries, particularly in remote regions from which many of our Russian sites recruited subjects. Therefore, it is possible that the frequent study visits had an unintended consequence for Russian subjects. The potential remedial benefits extend predominantly to cognition and negative symptoms, but could extend to other functional measures as well.
It may be concluded that some evidence of a procognitive effect with ABT-126 exists in US subjects. This effect was not of the magnitude observed in a previous study, wherein the Cohen's d effect size in the nonsmoking subgroup was $\sim 0.80$ (Haig et al, 2016). Subgroup analyses can be misleading, particularly when the sample size is reduced substantially. We believe ABT-126 has procognitive activity, but the magnitude of the effect is insufficient to detect in a large, multicenter trial where the intraindividual variability is too high to overcome. None of the additional exploratory analyses revealed a subset of the patient population that would be more likely to respond.

The higher doses used in this study did not appear to have a meaningful impact on the end points, in contrast to the previous study in which a monotonic dose-response was observed with ABT-126 in range of 10 to $25 \mathrm{mg}$ once daily (Haig et al, 2016). Some have argued that lower rates of receptor occupancy or a 'sweet spot' on the receptor occupancy curve is needed for $\alpha 7$ agonists (Andersen et al, 2013). Receptor occupancy studies have not been performed with ABT-126. However, given that the $25 \mathrm{mg}$ dose that showed efficacy in subgroup analyses in the prior study was re-evaluated in this study, missing the correct dose of ABT-126 is highly unlikely.

A trend toward significance was observed on the negative symptom scale, the NSA-16. A negative symptom treatment effect has been noted with other $\alpha 7$ agonists, including RG3487 (Umbricht et al, 2014), TC5619 (Lieberman et al, 2013), and EVP6124/encenicline (Keefe et al, 2015). Although the NSA-16 findings are hypothesis generating, the magnitude of change calls into question the importance of the results. Some experts believe a minimum of a 5-point difference is needed to be clinically relevant. Hence, the approximate 2-point difference between active treatment and 
placebo on the NSA-16 may not be clinically meaningful. In addition, in an exploratory subgroup analysis, the greatest treatment effects were observed in the subset of patients with lower (below the median) baseline negative symptom scores. Subjects with baseline scores greater than the median had a much greater placebo response. Nonetheless, a statistically significant difference was observed on the global measure, which could be an indicator of clinical relevance.

ABT-126 had an acceptable safety profile in subjects with cognitive impairment associated with schizophrenia in this study at doses up to $75 \mathrm{mg}$ daily. Constipation has been reported with other $\alpha 7$ agents and indeed was one of the more commonly reported adverse events in the companion smoking study (Haig et al, 2015). In this study, both diarrhea and constipation were reported at numerically greater frequencies with active treatment than with placebo, but both rates were low $(<5 \%)$.

In conclusion, ABT-126 did not demonstrate a consistent effect on cognition in nonsmoking subjects with schizophrenia; however, a trend toward an effect was observed on negative symptoms.

\section{FUNDING AND DISCLOSURE}

All authors are employees of AbbVie and hold AbbVie stock and/or stock options. GH and AAO are also AbbVie patent holders. This work was funded by AbbVie Inc. AbbVie participated in the study design, research, data collection, analysis and interpretation of data, writing, reviewing and approving the publication. Muriel Cunningham, MEd, of Winter Count Productions LLC, and Crystal Murcia, PhD, of The JB Ashtin Group, Inc., provided medical writing assistance compensated by AbbVie in the development of this publication.

\section{ACKNOWLEDGMENTS}

The authors wish to thank Earle E. Bain, MD of AbbVie for his contributions to the study.

\section{REFERENCES}

Agid O, Siu CO, Potkin SG, Kapur S, Watsky E, Vanderburg D et al (2013). Meta-regression analysis of placebo response in antipsychotic trials, 1970-2010. Am J Psychiatry 170: 1335-1344.

AhnAllen CG, Nestor PG, Shenton ME, McCarley RW, Niznikiewicz MA (2008). Early nicotine withdrawal and transdermal nicotine effects on neurocognitive performance in schizophrenia. Schizophr Res 100: 261-269.

Alphs LD, Summerfelt A, Lann H, Muller RJ (1989). The negative symptom assessment: a new instrument to assess negative symptoms of schizophrenia. Psychopharmacol Bull 25: 159-163.

Andersen N, Corradi J, Sine SM, Bouzat C (2013). Stoichiometry for activation of neuronal $\alpha 7$ nicotinic receptors. Proc Natl Acad Sci U S A 110: 20819-20824.

Barnes T (1989). A rating scale for drug-induced akathisia. British Journal of Pharmacology 154: 672-676.

Bowie CR, Harvey PD (2005). Cognition in schizophrenia: impairments, determinants, and functional importance. Psychiatr Clin North Am 28: 626.

Buchanan RW (2006). Important steps in the development of cognitive-enhancing drugs in schizophrenia. Am J Psychiatry 163: $1867-1869$.
Buchanan RW, Davis M, Goff D, Green MF, Keefe RS, Leon AC et al (2005). A summary of the FDA-NIMH-MATRICS workshop on clinical trial design for neurocognitive drugs for schizophrenia. Schizophr Bull 31: 5-19.

de Leon J, Diaz FJ (2005). A meta-analysis of worldwide studies demonstrates an association between schizophrenia and tobacco smoking behaviors. Schizophr Res 76: 135-157.

Dold M, Kasper S (2015). Increasing placebo response in antipsychotic trials: a clinical perspective. Evid Based Ment Health 18: 77-79.

Freedman R, Hall M, Adler LE, Leonard S (1995). Evidence in postmortem brain tissue for decreased numbers of hippocampal nicotinic receptors in schizophrenia. Biol Psychiatry 38: 22-33.

Freedman R, Olincy A, Buchanan RW, Harris JG, Gold JM, Johnson L et al (2008). Initial phase 2 trial of a nicotinic agonist in schizophrenia. Am J Psychiatry 165: 1040-1047.

Granholm E, Link P, Fish S, Kraemer H, Jeste D (2010). Age-related practice effects across longitudinal neuropsychological assessments in older people with schizophrenia. Neuropsychology 24: 616-624.

Green MF, Kern RS, Heaton RK (2004). Longitudinal studies of cognition and functional outcome in schizophrenia: implications for MATRICS. Schizophr Res 72: 41-51.

Guan ZZ, Zhang X, Blennow K, Nordberg A (1999). Decreased protein level of nicotinic receptor alpha7 subunit in the frontal cortex from schizophrenic brain. Neuroreport 10: 1779-1782.

Guy W (1976). ECDEU assessment manual for psychopharmacology, revised United States Department of Health, Education, and Welfare: Bethesda MD. Vol Publication ADM 76-338.

Haig GM, Bain EE, Robieson WZ, Baker JD, Othman AA (2016). A randomized trial to assess the efficacy and safety of ABT-126, a selective $\alpha 7$ nicotinic acetylcholine receptor agonist, in the treatment of cognitive impairment in schizophrenia. Am J Psychiatry; e-pub ahead of print 4 March 2016. doi:10.1176/ appi.ajp.2015.15010093.

Haig GM, Zhao J, Bain EE. (2015). Efficacy and safety of ABT-126, an $\alpha 7$ nicotinic cholinergic agonist, in treatment of cognitive impairment associated with schizophrenia: results from a phase $2 \mathrm{~b}$ study in smokers. Presented at the 15 th International Congress on Schizophrenia Research; March 28 - April 1, 2015; Colorado Springs, Colorado.

Harris JG, Kongs S, Allensworth D, Martin L, Tregellas J, Sullivan B et al (2004). Effects of nicotine on cognitive deficits in schizophrenia. Neuropsychopharmacology 29: 1378-1385.

Kay S, Fiszbein A, Opler L (1987). The Positive and Negative Syndrome Scale (PANSS) for schizophrenia. Schizophrenia Bulletin 13: 261-276.

Keefe RS, Eesley CE, Poe MP (2005). Defining a cognitive function decrement in schizophrenia. Biol Psychiatry 57: 688-691.

Keefe RS, Meltzer HA, Dgetluck N, Gawryl M, Koenig G, Moebius HJ et al (2015). Randomized, double-blind, placebocontrolled study of encenicline, an alpha-7 nicotinic acetylcholine receptor agonist as a treatment for cognitive impairment in schizophrenia. Neuropsychopharmacology 40: 3053-3060.

Keefe RS, Poe M, Walker TM, Kang JW, Harvey PD (2006). The Schizophrenia Cognition Rating Scale: an interview-based assessment and its relationship to cognition, real-world functioning, and functional capacity. Am J Psychiatry 163: 426-432.

Kern RS, Nuechterlein KH, Green MF, Baade LE, Fenton WS, Gold JM et al (2008). The MATRICS Consensus Cognitive Battery, part 2: co-norming and standardization. Am J Psychiatry 165: 214-220.

Kontis D, Huddy V, Reeder C, Landau S, Wykes T (2013). Effects of age and cognitive reserve on cognitive remediation therapy outcome in patients with schizophrenia. Am J Geriatr Psychiatry 21: $218-230$.

Levin ED, Rezvani AH (2007). Nicotinic interactions with antipsychotic drugs, models of schizophrenia and impacts on cognitive function. Biochem Pharmacol 74: 1182-1191. 
Lieberman JA, Dunbar G, Segreti AC, Girgis RR, Seoane F, Beaver JS et al (2013). A randomized exploratory trial of an alpha-7 nicotinic receptor agonist (TC-5619) for cognitive enhancement in schizophrenia. Neuropsychopharmacology 38: 968-975.

Myers CS, Robles O, Kakoyannis AN, Sherr JD, Avila MT, Blaxton TA et al (2004). Nicotine improves delayed recognition in schizophrenic patients. Psychopharmacology (Berl) 174: 334-340.

Nuechterlein KH, Green MF, Kern RS, Baade LE, Barch DM, Cohen JD et al (2008). The MATRICS Consensus Cognitive Battery, part 1: test selection, reliability, and validity. Am J Psychiatry 165: 203-213.

Olincy A, Harris JG, Johnson LL, Pender V, Kongs S, Allensworth D et al (2006). Proof-of-concept trial of an alpha7 nicotinic agonist in schizophrenia. Arch Gen Psychiatry 63: 630-638.

Patterson T, Goldman S (2005). The UCSD PerformanceBased Skills Assessment (UPSA-2). Copy written material R-10-06.
Posner K, Brown GK, Stanley B, Brent DA, Yershova KV, Oquendo MA et al (2011). The Columbia-Suicide Severity Rating Scale: initial validity and internal consistency findings from three multisite studies with adolescents and adults. Am J Psychiatry 168: 1266-1277.

Rickels K, Garcia-Espana F, Mandos LA, Case GW (2008). Physician Withdrawal Checklist (PWC-20). J Clin Psychopharmacol 28: 447-451.

Simpson G, Angus J (1970). A rating scale for extrapyramidal side effects. Acta Psychiatrica Scandinavica 212: 11-19.

Smith RC, Warner-Cohen J, Matute M, Butler E, Kelly E, Vaidhyanathaswamy S et al (2006). Effects of nicotine nasal spray on cognitive function in schizophrenia. Neuropsychopharmacology 31: 637-643.

Umbricht D, Keefe RS, Murray S, Lowe DA, Porter R, Garibaldi G et al (2014). A randomized, placebo-controlled study investigating the nicotinic alpha7 agonist, RG3487, for cognitive deficits in schizophrenia. Neuropsychopharmacology 39: 1568-1577.

Supplementary Information accompanies the paper on the Neuropsychopharmacology website (http://www.nature.com/npp) 